\title{
A New Flavonoid from Selaginella tamariscina (Beauv.) Spring
}

\author{
Jian-Feng Liu, ${ }^{a, c}$ Kang-Ping Xu, ${ }^{a}$ Fu-Shuang Li, ${ }^{a}$ Jian Shen, ${ }^{a}$ Chang-Ping Hu, ${ }^{a}$ Hui Zou, ${ }^{a}$ Fan Yang, ${ }^{a}$ \\ Guo-Ru LiU, ${ }^{a}$ Hong-Lin XIANG, ${ }^{a}$ Ying-Jun Zhou, ${ }^{a}$ Yuan-Jian LI, ${ }^{a}$ and Gui-Shan TAN ${ }^{*}, a, b$ \\ ${ }^{a}$ School of Pharmaceutical Sciences, Central South University; Changsha, Hunan 410013, China: ${ }^{b}$ Xiangya Hospital of \\ Central South University; Changsha, Hunan 410008, China: and ${ }^{c}$ Department of Pharmacy, The Third Affiliated People's \\ Hospital of Huaihua Medical College; Huaihua, Hunan 418000, China. \\ Received September 8, 2009; accepted December 27, 2009; published online January 14, 2010
}

A new flavonoid, 6-(2-hydroxy-5-carboxyphenyl)-apigenin (1), together with two new natural products, 3(4-hydroxyphenyl)-6,7-dihydroxy coumarin (2), 1-methoxy-3-methylanthraquinone (3) and four known compounds, were isolated from Selaginella tamariscina (BEauv.) Spring. The structures of the new isolated compounds were elucidated on the basis of 1D and 2D NMR as well as ESI-HR-MS spectroscopic analysis.

Key words Selaginella tamariscina; Selaginellaceae; flavonoid; coumarin; anthraquinone

Selaginella tamariscina (Beauv.) Spring, one species from genus Selaginella, has been introduced in Chinese Pharmacopeia for the effectiveness of promoting blood circulation. Studies on the chemical constituents of Selaginella led to the discovery of many compounds including biflavonoids, phenylpropanoids, anthraquinones, steroids, alkaloids and organic acids. Recently, some unusual natural pigments with novel carbon framework named selaginellin, selaginellin A and selaginellin B were isolated from $S$. sinensis ${ }^{1)}$ and $S$. tamariscina. ${ }^{2)}$ Previously, we have isolated selaginellin $\mathrm{C}$, the fourth novel unusual natural pigments from $S$. pulvinata Maxim. (Hook et Grev.) ${ }^{3)}$ and a new flavonoid from $S$. tamariscina. ${ }^{4)}$ As a continuation of our work, another new flavonoid, 6-(2-hydroxy-5-carboxyphenyl)-apigenin (1) was isolated from the $75 \%(\mathrm{v} / \mathrm{v})$ ethanol extract of S. tamariscina together with two new natural products, 3-(4-hydroxyphenyl)-6,7-dihydroxy coumarin (2), ${ }^{5)}$ 1-methoxy-3-methylanthraquinone $^{6}$ (3) and four known compounds heveaflavone (4), amentoflavone (5), heptadecanoic acid (6) and $\beta$-sitosterol (7).

\section{Results and Discussion}

Compound 1 was obtained as amorphous yellow powder. The molecular formula, $\mathrm{C}_{22} \mathrm{H}_{14} \mathrm{O}_{8}$, was determined on the basis of positive electron spray ionization mass spectrometry (ESI-MS) $\left(\mathrm{m} / \mathrm{z} 407.0[\mathrm{M}+\mathrm{H}]^{+}\right)$and HR-ESI-MS data $[\mathrm{m} / \mathrm{z}$ : 407.07851 $[\mathrm{M}+\mathrm{H}]^{+}$(Calcd for $\left.\left.\mathrm{C}_{22} \mathrm{H}_{15} \mathrm{O}_{8}, 407.07669\right)\right]$. Its UV spectrum showed the maximum absorption at 339,253 and $223 \mathrm{~nm}$. IR spectrum showed the presence of hydroxyl $\left(3432.0 \mathrm{~cm}^{-1}\right)$, conjugated carbonyl $\left(1654.4 \mathrm{~cm}^{-1}\right)$, and aromatic ring $\left(1607.4,1579.0,1510.1 \mathrm{~cm}^{-1}\right)$. Examination of the ${ }^{1} \mathrm{H}$ - and ${ }^{13} \mathrm{C}-\mathrm{NMR}$ of compound $\mathbf{1}$ and comparison to the corresponding signals in 6-(2-hydroxy-5-acetylphenyl)-apigenin reported previously ${ }^{4}$ indicated that compound $\mathbf{1}$ had similar structure with 6-(2-hydroxy-5-acetylphenyl)-apigenin. As evident from the ${ }^{1} \mathrm{H}-\mathrm{NMR}$ spectrum, five hydroxyl groups were exhibited at $\delta 13.09(1 \mathrm{H}, \mathrm{s}, 5-\mathrm{OH}), \delta 12.49$ $(1 \mathrm{H}$, br s, 5"-COOH), $\delta 10.74(1 \mathrm{H}, \mathrm{br}), \delta 10.32(1 \mathrm{H}, \mathrm{s})$ and $\delta 10.21(1 \mathrm{H}$, brs $)$. An $\mathrm{AA}^{\prime} \mathrm{XX}^{\prime}$ coupling system signals at $\delta$ $7.55\left(2 \mathrm{H}, \mathrm{d}, J=8.8 \mathrm{~Hz}, \mathrm{H}-2^{\prime}, 6^{\prime}\right)$ and $\delta 6.76(2 \mathrm{H}, \mathrm{d}$, $\left.J=8.8 \mathrm{~Hz}, \mathrm{H}-3^{\prime}, 5^{\prime}\right)$ indicated the para-substitution of ring B. In heteronuclear multiple bond coherence (HMBC) spectrum, the correlation peaks between $\delta 6.80(\mathrm{H}-3)$ and $\delta$
$163.9(\mathrm{C}-2), \delta 182.4(\mathrm{C}-4), \delta 121.4\left(\mathrm{C}-1^{\prime}\right)$ suggested that $\mathrm{C}$ 3 was not be substituted (Fig. 2). Due to the ${ }^{1} \mathrm{H}-{ }^{1} \mathrm{H}$ correlation spectroscopy (COSY) and HMBC spectrum, three aromatic protons $\delta 7.87\left(1 \mathrm{H}\right.$, dd, $\left.J=8.4,2.0 \mathrm{~Hz}, \mathrm{H}-4^{\prime \prime}\right), \delta 7.79$ $\left(1 \mathrm{H}, \mathrm{d}, J=2.0 \mathrm{~Hz}, \mathrm{H}-6^{\prime \prime}\right)$ and $\delta 7.06\left(1 \mathrm{H}, \mathrm{d}, J=8.4 \mathrm{~Hz}, \mathrm{H}-3^{\prime \prime}\right)$ should belong to ring D. The HMBC correlations between $\delta$ $167.5\left(5^{\prime \prime}-\mathrm{COOH}\right)$ and $\delta 7.87\left(\mathrm{H}-4^{\prime \prime}\right), \delta 7.79\left(\mathrm{H}-6^{\prime \prime}\right)$ indicated that the carboxy group was located at C- $5^{\prime \prime}$. The HMBC correlations between $\delta 160.2\left(\mathrm{C}-2^{\prime \prime}\right)$ and $\delta 7.87\left(\mathrm{H}-4^{\prime \prime}\right), \delta 7.79$ $\left(\mathrm{H}-6^{\prime \prime}\right)$ indicated a hydroxyl group was located at C-2". HMBC correlations between $\delta 103.9$ (C-6) and $\delta 7.79\left(\mathrm{H}-6^{\prime \prime}\right)$ concluded the linkage of the carboxyphenyl was located at the C- 6 position of ring A. On the basis of above evidences, compound 1 was determined to be 3-(2-(4-hydroxyphenyl)5,7-dihydroxy-chromen-6-yl)-4-hydroxy-benzoic acid, named 6-(2-hydroxy-5-carboxyphenyl)-apigenin.

Compound 2 was also obtained as an amorphous yellow powder. The molecular formula, $\mathrm{C}_{15} \mathrm{H}_{10} \mathrm{O}_{5}$, was determined<smiles>O=C(O)c1ccc(O)c(-c2c(O)cc3oc(-c4ccc(O)cc4)cc(=O)c3c2O)c1</smiles><smiles></smiles><smiles>COc1cc(C)cc2c1C(=O)c1ccccc1C2=O</smiles>

Fig. 1. Structure of Compounds $\mathbf{1}-\mathbf{3}$ 

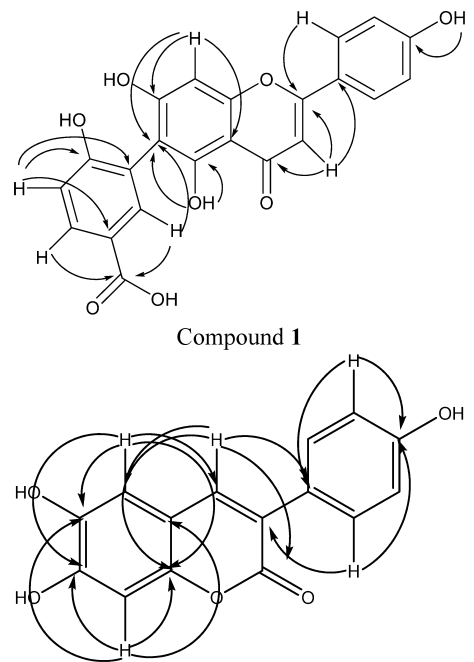

Compound 2

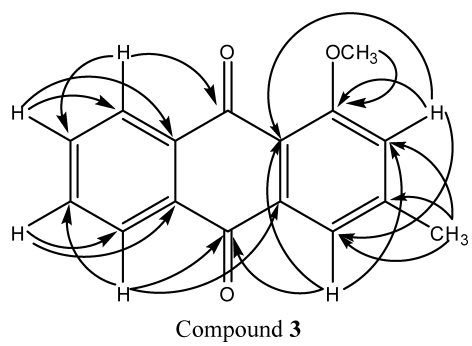

Fig. 2. Key HMBC Correlations of $\mathbf{1}-\mathbf{3}$

on the basis of positive ESI-MS $\left(m / z: 271.3[\mathrm{M}+\mathrm{H}]^{+}\right)$and HR-ESI-MS data $\left[\mathrm{m} / \mathrm{z}: 271.0596[\mathrm{M}+\mathrm{H}]^{+}\right.$(Calcd for $\left.\left.\mathrm{C}_{15} \mathrm{H}_{11} \mathrm{O}_{5}, 271.0606\right)\right]$. Its UV spectrum showed the maximum absorption at 274 and $220 \mathrm{~nm}$. Its IR spectrum showed typical absorptions of hydroxyl $\left(3433.8 \mathrm{~cm}^{-1}\right)$, carbonyl $\left(1716.5 \mathrm{~cm}^{-1}\right)$, and aromatic ring $\left(1584.9,1509.3 \mathrm{~cm}^{-1}\right)$. The structure of 2 was established from analysis of the ${ }^{1} \mathrm{H}$ - and ${ }^{13} \mathrm{C}$-NMR spectra, as shown in Table $1 .{ }^{13} \mathrm{C}$-NMR displayed 15 carbon signals, nine being typical for an esculetin (6,7-dihydroxy-coumarin), ${ }^{7)}$ the other 6 signals were ascribable to a para-substitution of phenyl moiety, which was verified by an $\mathrm{AA}^{\prime} \mathrm{XX}^{\prime}$ coupling system signals at $\delta 7.53(2 \mathrm{H}, \mathrm{d}, J=8.4 \mathrm{~Hz}$, $\left.\mathrm{H}-2^{\prime}, 6^{\prime}\right)$ and $\delta 6.80\left(2 \mathrm{H}, \mathrm{d}, J=8.4 \mathrm{~Hz}, \mathrm{H}-3^{\prime}, 5^{\prime}\right)$ exhibited in ${ }^{1} \mathrm{H}-\mathrm{NMR}$. The signal at $\delta 157.5$ showed that there was a hydroxyl located at C-4'. The HMBC correlations between $\delta$ $121.8(\mathrm{C}-3)$ and $\delta 7.53\left(\mathrm{H}-2^{\prime}, 6^{\prime}\right), \delta 126.4\left(\mathrm{C}-1^{\prime}\right)$ and $\delta 7.93$ (H-4) (Fig. 2) concluded the linkage of the $p$-hydroxyphenyl was located at the $\mathrm{C}-3$ position of esculetin.

Compound 3 was obtained as an yellow crystal $\left(\mathrm{CH}_{3} \mathrm{Cl}_{3} /\right.$ $\mathrm{MeOH})$, mp $183.7-185.3^{\circ} \mathrm{C}$, The molecular formula, $\mathrm{C}_{16} \mathrm{H}_{12} \mathrm{O}_{3}$, was determined on the basis of positive EI-MS $\left(\mathrm{m} / \mathrm{z}: 252[\mathrm{M}]^{+}\right)$, ESI-MS $\left(\mathrm{m} / z: 253.2[\mathrm{M}+\mathrm{H}]^{+}\right)$and HRESI-MS data $\left[\mathrm{m} / \mathrm{z}: 253.0856[\mathrm{M}+\mathrm{H}]^{+}\left(\right.\right.$Calcd for $\mathrm{C}_{16} \mathrm{H}_{13} \mathrm{O}_{3}$, 253.0865)]. Its UV spectrum showed the maximum absorption at 386,256 and $211 \mathrm{~nm}$. Its IR spectrum showed the presence of carbonyl $\left(1736.5 \mathrm{~cm}^{-1}\right)$ functionality. The structure of 3 was established from analysis of the ${ }^{1} \mathrm{H}$ - and ${ }^{13} \mathrm{C}$ NMR spectra, as shown in Table 2. The extensive NMR spectral analyses indicated an 1-methoxy-9,10-anthraquinone skeleton. ${ }^{8)}$ Besides, the location of a methyl ( $\delta$ 56.5) was identified by the correlations between $\delta 2.52\left(-\mathrm{CH}_{3}\right)$ and $\delta$ $118.5(\mathrm{C}-2), \delta 2.52\left(-\mathrm{CH}_{3}\right)$ and $\delta 120.6(\mathrm{C}-4)$ in HMBC ex-
Table 1. NMR Data for Compounds $\mathbf{1}$ and $\mathbf{2}$ in DMSO- $d_{6}$

\begin{tabular}{|c|c|c|c|c|}
\hline \multirow{2}{*}{ Position } & \multicolumn{2}{|r|}{1} & \multicolumn{2}{|c|}{2} \\
\hline & $\delta_{\mathrm{C}}^{a)}$ & $\delta_{\mathrm{H}}^{b)}(J$ in $\mathrm{Hz})$ & $\delta_{\mathrm{C}}^{a)}$ & $\delta_{\mathrm{H}}^{b)}(J$ in $\mathrm{Hz})$ \\
\hline 2 & 163.9 & - & 161.0 & - \\
\hline 3 & 102.8 & $6.80 \mathrm{~s}$ & 121.8 & - \\
\hline 4 & 182.4 & - & 139.6 & $7.93 \mathrm{~s}$ \\
\hline 5 & 161.3 & - & 111.7 & $6.99 \mathrm{~s}$ \\
\hline 6 & 103.9 & - & 143.8 & - \\
\hline 7 & 162.0 & - & 151.9 & - \\
\hline 8 & 98.9 & $6.39 \mathrm{~s}$ & 102.2 & $6.69 \mathrm{~s}$ \\
\hline 9 & 154.7 & - & 148.4 & - \\
\hline 10 & 104.3 & - & 111.2 & - \\
\hline $1^{\prime}$ & 121.4 & - & 126.4 & - \\
\hline $2^{\prime}$ & 128.5 & $7.55 \mathrm{~d}(8.8)$ & 129.7 & $7.53 \mathrm{~d}(8.4)$ \\
\hline $3^{\prime}$ & 116.1 & $6.76 \mathrm{~d}(8.8)$ & 115.2 & $6.80 \mathrm{~d}(8.4)$ \\
\hline $4^{\prime}$ & 160.7 & - & 157.5 & - \\
\hline $5^{\prime}$ & 116.1 & $6.76 \mathrm{~d}(8.8)$ & 115.2 & $6.80 \mathrm{~d}(8.4)$ \\
\hline $6^{\prime}$ & 128.5 & $7.55 \mathrm{~d}(8.8)$ & 129.7 & $7.53 \mathrm{~d}(8.4)$ \\
\hline $1^{\prime \prime}$ & 121.6 & - & - & - \\
\hline $2^{\prime \prime}$ & 160.2 & - & - & - \\
\hline $3^{\prime \prime}$ & 115.6 & $7.06 \mathrm{~d}(8.4)$ & - & - \\
\hline $4^{\prime \prime}$ & 131.0 & $7.87 \mathrm{dd}(8.4,2.0)$ & - & - \\
\hline $5^{\prime \prime}$ & 119.2 & - & - & - \\
\hline $6^{\prime \prime}$ & 134.9 & $7.79 \mathrm{~d}(2.0)$ & - & - \\
\hline $5-\mathrm{OH}$ & - & 13.09 br s & - & - \\
\hline $5^{\prime \prime}-\mathrm{COOH}$ & 167.5 & 12.49 br s & - & - \\
\hline
\end{tabular}

${ }^{a)}$ Recorded at $100 \mathrm{MHz}$. ${ }^{\text {b) }}$ Recorded at $400 \mathrm{MHz}$.

Table 2. NMR Data for Compound 3 in $\mathrm{CDCl}_{3}$

\begin{tabular}{|c|c|c|}
\hline \multirow{2}{*}{ Position } & \multicolumn{2}{|c|}{3} \\
\hline & $\delta_{\mathrm{C}}^{a)}$ & $\delta_{\mathrm{H}}^{b)}(J$ in $\mathrm{Hz})$ \\
\hline 1 & 160.6 & - \\
\hline 2 & 118.5 & 7.15 br s \\
\hline 3 & 146.5 & - \\
\hline 4 & 120.6 & 7.79 br s \\
\hline $4 a$ & 135.5 & - \\
\hline 5 & 126.5 & $8.22 \mathrm{dd}(7.5,1.0)$ \\
\hline 6 & 134.2 & $7.77 \mathrm{dt}(7.5,1.0)$ \\
\hline 7 & 133.1 & $7.73 \mathrm{dt}(7.5,1.0)$ \\
\hline 8 & 127.2 & $8.28 \mathrm{dd}(7.5,1.0)$ \\
\hline $8 \mathrm{a}$ & 132.5 & - \\
\hline 9 & 182.3 & - \\
\hline $9 a$ & 119.3 & - \\
\hline 10 & 183.8 & - \\
\hline $10 \mathrm{a}$ & 135.1 & - \\
\hline $1-\mathrm{OCH}_{3}$ & 56.5 & $4.05 \mathrm{~s}$ \\
\hline $3-\mathrm{CH}_{3}$ & 22.4 & $2.52 \mathrm{~s}$ \\
\hline
\end{tabular}

a) Recorded at $125 \mathrm{MHz}$. b) Recorded at $500 \mathrm{MHz}$.

periments (Fig. 2). The assignment of NMR data to compound 3 was further comfirmed by means of heteronuclear single quantum coherence (HSQC) and HMBC experiments.

\section{Experimental}

General Experimental Procedures Melting points were measured on Buchi-540 melting point apparatus (uncorrected); UV spectra were performed on a Shimadzu UV-2450 instrument (Japan); IR spectra were obtained on a Nicolet Avatar (U.S.A.) 360 FT-IR instrument as a film on $\mathrm{KBr}$ disk; NMR spectra were recorded on a Varian (U.S.A.) INOVA-400 spectrometers and Bruker AV-500 spectrometer with tetramethylsilane (TMS) as internal standard; MS spectra were measured on an LCQ-Advantage (U.S.A.) mass spectrometer and Q-Trap LC/MS/MS System Turboinospray source (Applied Biosystems/MDS Sciex U.S.A.); HR-ESI-MS spectra were recorded on a Micromass Zabspec (U.K.) HR-MS spectrometer and JMS- 
T100 CS. Column was run using silica gel (200-300 mesh). The prepHPLC was performed on LC-8A (Shimadzu, pre-ODS, $20 \times 250 \mathrm{~mm}, 10 \mu \mathrm{m}$, UV detecter, $\left.\mathrm{MeOH}-\mathrm{H}_{2} \mathrm{O}\right)$.

Plant Material Herbs of $S$. tamariscina were collected in Jiangxi Province, P. R. China, and were identified by Associate Prof. Jin-Ping Li (Central South University, Changsha, P. R. China). A voucher specimen was deposited in School of Pharmaceutical Sciences, Central South University (No. JB-003).

Extraction and Isolation The air-dried sample of $S$. tamariscina $(14.0 \mathrm{~kg})$ was soaked twice with cold $75 \% \mathrm{EtOH}$. After removal of the solvent under reduced pressure, the extract $(1450 \mathrm{~g})$ was chromatographed over D-101 macroporous resin column with $\mathrm{EtOH} / \mathrm{H}_{2} \mathrm{O}(\mathrm{v} / \mathrm{v})$ gradient elution $(0$, $30,60,95 \%$ ). The $60 \% \mathrm{EtOH}$ portion was subject to a combination of chromatographies such as silica gel column chromatography, gel permeation chromatography and preparative HPLC to yield compound 1 (10.7 mg), 2 (7.8 mg), 3 (9.7 mg), 4 (107.3 mg), 5 (314.5 mg), 6 (52.6 mg), 7 (232.6 mg).

6-(2-Hydroxy-5-carboxyphenyl)-apigenin (1) $\mathrm{C}_{22} \mathrm{H}_{14} \mathrm{O}_{8}$ : Amorphous yellow powder; UV $\lambda_{\max }(\mathrm{MeOH}): 339,253,223 \mathrm{~nm}$; IR bands $(\mathrm{KBr}): 3432.0$, 1654.4, 1607.4, 1579.0, 1510.1 $\mathrm{cm}^{-1}$; ${ }^{1} \mathrm{H}-$ and ${ }^{13} \mathrm{C}-\mathrm{NMR}$ see Table 1; ESIMS $m / z$ : $407.0[\mathrm{M}+\mathrm{H}]^{+}$; ESI-HR-MS $m / z$ : $407.07851[\mathrm{M}+\mathrm{H}]^{+}$(Calcd for $\left.\mathrm{C}_{22} \mathrm{H}_{15} \mathrm{O}_{8}\right), 407.07669$.

3-(4-Hydroxyphenyl)-6,7-dihydroxycoumarin (2) $\mathrm{C}_{15} \mathrm{H}_{10} \mathrm{O}_{5}$ : Yellow amorphous powder; ${ }^{1} \mathrm{H}$ - and ${ }^{13} \mathrm{C}-\mathrm{NMR}$ see Table 1 ; UV $\lambda_{\max }(\mathrm{MeOH}) \mathrm{nm}: 274$, 220; IR (KBr) cm $\mathrm{cm}^{-1}$ : 3433.8, 1716.5, 1584.9, 1509.3; EI-MS m/z: 288.3 $\left[\mathrm{M}+\mathrm{H}_{2} \mathrm{O}\right]^{+}$, ESI-MS $\left(\mathrm{m} / \mathrm{z}: 271.3[\mathrm{M}+\mathrm{H}]^{+}\right)$and HR-ESI-MS data $[\mathrm{m} / \mathrm{z}$ : $271.0596[\mathrm{M}+\mathrm{H}]^{+}$(Calcd for $\left.\left.\mathrm{C}_{15} \mathrm{H}_{11} \mathrm{O}_{5}, 271.0606\right)\right]$.

1-Methoxy-3-methylanthraquinone (3) $\mathrm{C}_{16} \mathrm{H}_{12} \mathrm{O}_{3}$ : Yellow amorphous powder; mp 183.7-185.3 ${ }^{\circ} \mathrm{C}$; EI-MS m/z: 252 [M] $^{+}, 237,209,206,178$; ESIMS $\left(m / z: 253.2[\mathrm{M}+\mathrm{H}]^{+}\right)$and HR-ESI-MS data $\left[m / z: 253.0856[\mathrm{M}+\mathrm{H}]^{+}\right.$ (Calcd for $\left.\mathrm{C}_{16} \mathrm{H}_{13} \mathrm{O}_{3}, 253.0865\right)$ ]; ${ }^{1} \mathrm{H}$ - and ${ }^{13} \mathrm{C}$-NMR see Table 2; IR (KBr) $\mathrm{cm}^{-1}$ : 2953.4, 2849.5, 1736.6, 1457.3, 1397.7, 1384.9.

Identification of compounds $\mathbf{4}, \mathbf{5}$ were performed by comparing NMR data with those reported in the literature. ${ }^{9,10)}$ Identification of compounds $\mathbf{6}$,
7 were supported by TLC comparison with authentic compounds.

Acknowledgment This project was supported by the National Natural Science Foundation of China (No. 30873149), the S \& T Project of Hunan Provincial Science \& Technology Department (No. 2008FJ4182), Traditional Chinese Medicine Research Program of Hunan Province (No. 2009059), University Students Innovation Test Project of Ministry of Education of the People's Republic of China (No. YA09060), and Precision Equipment and Apparatus Foundation of Central South University (No. ZKJ2009019).

\section{References and Notes}

1) Zhang L. P., Liang Y. M., Wei X. C., Cheng D. L., J. Org. Chem., 72, 3921-3924 (2007)

2) Cheng X. L., Ma S. C., Yu J., Yu J. D., Yang S. Y., Xiao X. Y., Hu J. Y., Lu Y., Haw P. C., But P. P.-H., Lin R. C., Chem. Pharm. Bull., 56, 982-984 (2008).

3) Tan G S., Xu K. P., Li F. S., Wang C. J., Li T. Y., Hu C. P., Shen J., Zhou Y. J., Li Y. J., J. Asian Nat. Prod. Res., 11, 1001-1004 (2009).

4) Liu J. F., Xu K. P., Jiang D. J., Li F. S., Shen J., Zhou Y. J., Xu P. S., Tan B., Tan G. S., Chin. Chem. Lett., 20, 595-597 (2009).

5) Bulut M., Chim. Acta Turc., 19, 17-26 (1992).

6) Donald W. C., Geoffrey I. F., Antonio F. P., Aus. J. Chem., 30, 1255 1258 (1977).

7) AIST: Integrated Spectral Database System of Organic Compounds. (Data were obtained from the National Institute of Advanced Industrial Science and Technology (Japan).)

8) Berger Y., Berger-Deguée M., Castonguay A., Org. Mag. Res., 15, 303 (1981).

9) Markham K. R., Sheppard C., Geiger H., Phytochemistry, 26, 3335 (1987).

10) Lin R. C., Skaltsounis A.-L., Seguin E., Tillequin F., Koch M., Planta Med., 60, 168 (1994). 